\title{
On the performance of energy harvesting AF partial relay selection with TAS and outdated channel state information over identical channels
}

\author{
Kehinde O. Odeyemi ${ }^{1}$, Pius A. Owolawi ${ }^{2}$ \\ ${ }^{1}$ Department of Electrical and Electronic Engineering, Faculty of Technology, University of Ibadan, Nigeria \\ ${ }^{2}$ Department of Computer Systems Engineering, Tshwane University of Technology, South Africa
}

\begin{tabular}{l} 
Article Info \\
\hline Article history: \\
Received Apr 27, 2019 \\
Revised Apr 7, 2020 \\
Accepted Apr 20, 2020 \\
\hline
\end{tabular}

Keywords:

Channel state information

Energy harvesting

Maximum ratio combining

Partial relay selection

Transmit antenna selection

\begin{abstract}
Energy scarcity has been known to be one of the most noticeable challenges in wireless communication system. In this paper, the performance of an energy harvesting based partial relay selection (PRS) cooperative system with transmit antenna selection (TAS) and outdated channel state information (CSI) is investigated. The system dual-hops links are assumed to follow Rayleigh distribution and the relay selection is based on outdated CSI of the first link. To realize the benefit of multiple antenna, the amplified-andforward (AF) relay nodes then employs the TAS technique for signal transmission and signal reception is achieved at the destination through maximum ratio combining (MRC) scheme. Thus, the closed-form expression for the system equivalent end-to-end cumulative distribution function (CDF) is derived. Based on this, the analytical closed-form expressions for the outage probability, average bit error rate, and throughput for the delaylimited transmission mode are then obtained. The results illustrated that the energy harvesting time, relay distance, channel correlation coefficient, the number of relay transmit antennas and destination received antenna have significant effect on the system performance. Monte-carol simulation is employed to validate the accuracy of the derived expressions.
\end{abstract}

Copyright $@ 2020$ Institute of Advanced Engineering and Science. All rights reserved.

\section{Corresponding Author:}

Kehinde O. Odeyemi,

Department of Electrical and Electronic Engineering,

Factulty of Technology, University of Ibadan,

Ibadan, Nigeria.

Email: odeyemikenny@gmail.com

\section{INTRODUCTION}

Traditionally, relay nodes within the wireless cooperative network are usually equipped with powerlimited batteries that are infeasible, costly and even impossible to replace in some applications $[1,2]$. As a result of this, energy harvesting $(\mathrm{EH})$ has been considered as a promising solution to alleviate this stated energy constraint in wireless system. Therefore, research studies have suggested that batteries can be wirelessly recharge using external power sources from free ambient such as solar, wind, thermal and vibration [3]. These conventional sources are not always available and mostly suffers from time-varying problem and materials inefficiency $[4,5]$. Based on this, RF source has been regarded as the best alternative means of harvesting energy in wireless communication since it carries both the energy and information at the same time without monitoring and maintenance [6]. In this case, two schemes have been suggested in literature through which energy can be harvested and this include time switching (TS) and power splitting (PS) protocols. In TS protocol, the transmission time is divided into two parts where the receiver nodes spend one to harvest energy from the source and the remaining for information transmission. In PS protocol on the other hand, the receiver nodes used part of the EH and the other for information processing [7]. 
The deployment of relays in wireless networks can extend the signal coverage and overcoming the channel impairment as a result of fading [8]. Therefore, two strategies have been suggested for relay to transmit its received signal to the destination for which include decode-and-forward (DF) and amplify-andforward (AF) of relaying system [4, 9]. In DF relaying system, the received signal is fully decoded and the decoded copy is retransmitted to the destination. In AF relaying system on the other hand, the received signal is simply amplified and forwarded to the destination without decoding leading to its simplicity and effective implementation cost [10]. In the wireless cooperative system with multiple relays, numerous relay selection techniques have proposed [6] and this include the partial relay selection where selection of relays depends on the CSI of the source-relay links or relay-destination links. This thus avoids the issues such as high power consumption, overhead and network delays, and can also prolong the network lifetime [11, 12]. Unlike the best and opportunistic relay selection approaches, which require global knowledge of the CSI of each of the two hops $[12,13]$ leading to time synchronization, continuous channel feedback and high power consumption.

Reducing the energy consumption of cooperative relaying system through the energy harvesting has drawn more attention in the research community has attracted attention in the research community. The performance of dual-hop relaying network with three different wireless EH mechanisms at the DF relay nodes over the generalized channels was studied [14]. In [15], the performance of a wireless information power transfer in AF relay network with multiple antenna over dissimilar channel and the benefit of MRC/TAS was exploit for the relay node was investigated. The Ergodic outage and capacity performance of wireless power transfer based AF relaying system was presented in [16]. Also, the performance of DF-based EH with the source node and the destination employing TAS and MRC respectively was evaluated in [17]. The throughput performance of an AF relay based EH under delay-tolerance and limited transmission modes was investigated in [7] over Rayleigh fading channels. However, all these aforementioned works are limited to a single relay-based EH cooperative systems.

In case of multiple relay selection, the performance of EH cooperative system with optimal relay selection under the CSI and energy side information was investigated in [18]. In addition, incremental relay network with EH-based relay selection was proposed in [19] where the selected relay harvesting the largest source energy was chosen to forward the information to the destination. In [20], the performance of AF relaying energy harvesting system with $k$ th best PRS and energy beamforming over Nakagami-m was evaluated. In this work, it was assumed that both the source and the destination are multiple antenna nodes and the relay are single antenna nodes. In all these aforementioned studies, perfect CSI was assumed for the system. Motivated by this, the performance of the PRS-based EH system under the TAS/MRC is investigated in this paper where the relay nodes employ the TAS technique at the relay nodes and maximum ratio combining (MRC) scheme at the destination. The exact closed-form expression for the system outage probability and average bit error rate are obtained. Utilizing the outage probability, the throughput for the system is obtained based on delay-limited transmission mode. The impact of energy harvesting time, relay distance, channel correlation coefficient, the number of relay transmit antennas and destination received antenna on the concerned system are demonstrated.

\section{SYSTEM AND CHANNEL MODEL}

In this paper, a dual-hops PRS based energy harvesting system with PRS and outdated CSI is presented in Figure 1 (a). The source (S) is equipped with a single transmit antenna and the destination (D) has multiple receive antenna $N_{D}$. The relay (R) of $N$ nodes utilize a single receive antenna and multiple transmit antenna $N_{R}^{t}$. It is assumed that the S-to-R and R-to-D links undergo Rayleigh fading distribution without direct link between the source and the destination. The relay nodes employ TAS scheme to select the best antenna to forward the signal to the destination through which the instantaneous SNR at the destination is maximized. At the destination, MRC is used to combine the transmitted signal from the relay node(s). As a result of channel fast fading, it is considered that relay selection is based on outdated CSI. Thus, the instantaneous SNR of the first link and the one used for PRS are two correlated random variables with channel correlation coefficient $\rho$.The transmission over the links is assumed to be in half-duplex mode so as to avoid inter-signal interference. Therefore, the overall systems communication is established in two different phases.

During the first phase, the communication between the $\mathrm{S}$ and $\mathrm{R}$ nodes is divided into power transmission and information transmission as presented in Figure 1(b). Based on this, the $k-t h$ selected relay(s) harvest the energy and then receive the information from the source using the time switching-base relay (TSR) protocol. Thereafter, the source information is amplified through AF relaying and then convey to the destination using TAS technique. In TSR protocol, a total block time slot of $T$ is divided into three parts: for energy harvesting as $\alpha T$, information transmission between $\mathrm{S}$-to-R as $(1-\alpha) T / 2$ and information 
transmission between R-to-D as $(1-\alpha) T / 2$. The parameter $\alpha$ denotes the energy having time factor at $0 \leq \alpha \leq 1$. Thus, the receive signal by the $k-t h$ selected relay(s) can be expressed as:

$$
y_{1(k)}(t)=\sqrt{\frac{P_{S}}{d_{1}^{\vartheta_{1}}}} h_{1(k)} x(t)+n_{R(k)}(t)
$$

where $P_{S}$ is the source transmitted power, $h_{1(k)}$ is $k-t h$ the channel gain, $d_{1}$ is the distance between the source and the relay, $\vartheta_{1}$ denotes the path loss exponent, $x(t)$ represents the sources information and $n_{R(k)}$ is the AWGN at the $k-t h$ selected relay(s) with zero mean and variance $\sigma_{1}^{2}$. The energy harvested by an energy receiver at the $k-t h$ selected relay(s) over the time $\alpha T$ can be expressed as $[1,11]$ :

$$
E_{h, k}=\frac{\eta \alpha T\left|h_{1(k)}\right|^{2}}{d_{1}^{\vartheta_{1}}}
$$

where $0 \leq \eta \leq 1$ denotes the energy harvesting efficiency which depends on the rectification process and the circuitry.

During the second phase, the remaining time slot of $(1-\alpha) T / 2$ duration is then used by the $k-t h$ selected relay(s) to amplifies and retransmit the information to the destination using TAS principle. The destination thus employs MRC technique to combine the transmitted signal from the $k-t h$ selected relay(s) and the received signal can be expressed as:

$$
y_{2}(t)=\sqrt{\frac{P_{R(k)}}{d_{2}^{\vartheta_{2}}}} h_{2(k)} \hat{x}(t)+n_{D}(t)
$$

where $h_{2(k)}$ the channel gain, $d_{2}$ is the distance between the relay and destination, $\vartheta_{2}$ denotes the path loss exponent, $\hat{x}(t)$ represents the decoded information of $x(t), n_{D}$ is the AWGN at the destination with zero mean and variance $\sigma_{2}^{2}$, and $P_{R(k)}$ is the $k-t h$ selected relay(s) transmit power obtained from energy harvesting for relaying amplification and this can be expressed as [14]:

$$
P_{R(k)}=\frac{E_{h(k)}}{(1-\alpha) T / 2} \triangleq \frac{2 \eta \alpha P_{S}\left|h_{1(k)}\right|^{2}}{(1-\alpha) d_{1}^{\vartheta_{1}}}
$$

Since the relays are fixed-gain AF nodes, the equivalent end-to-end instantaneous SNR at the destination for the system can be approximately expressed by following the same approach in [11] as:

$$
\gamma_{e q}=\frac{\phi \widetilde{\gamma}_{1(k)} \gamma_{2(k)}}{\psi \gamma_{2(k)}+1}
$$

where $\tilde{\gamma}_{1(k)}=\left|h_{1(k)}\right|^{2}, \gamma_{2(k)}=\left|h_{2(k)}\right|^{2}, \psi=\frac{2 \eta \alpha}{(1-\alpha) d_{1}^{\vartheta_{1}}}$, and $\phi=\frac{2 \eta \alpha \gamma_{T}}{(1-\alpha) d_{1}^{\vartheta_{1}} d_{1}^{\vartheta_{2}}}$ for $\gamma_{T}$ denotes the average transmit SNR.

In this study, the dual-hops links for the system are characterized by the Rayleigh fading and the probability density function (PDF) for the distribution for the S-to-R link under the partial relay selection system can be expressed as $[11,12]$ :

$$
\begin{aligned}
f_{\gamma_{1(k)}}(x) & =k\left(\begin{array}{l}
N \\
k
\end{array}\right) \sum_{n=0}^{k-1}\left(\begin{array}{c}
k-1 \\
n
\end{array}\right) \frac{(-1)^{n}}{((N-k+n)(1-\rho)+1) \Omega_{1}} \\
& \times \exp \left(-\frac{(N-k+p+1) x}{((N-k+n)(1-\rho)+1) \Omega_{1}}\right)
\end{aligned}
$$

where $\rho$ is the channel correlation coefficient, $\Omega_{1}=\mathbf{E}\left[\left|h_{1(k)}\right|^{2}\right]$

Also, the corresponding CDF can be obtained by integrating (6) as:

$$
\begin{aligned}
F_{\gamma_{1(k)}}(x) & =1-k\left(\begin{array}{l}
N \\
k
\end{array}\right) \sum_{n=0}^{k-1}\left(\begin{array}{c}
k-1 \\
n
\end{array}\right) \frac{(-1)^{n}}{((N-k+n)+1)} \\
& \times \exp \left(-\frac{(N-k+n+1) x}{((N-k+n)(1-\rho)+1) \Omega_{1}}\right)
\end{aligned}
$$


By the principle of TAS/MRC, the PDF for the R-to-D channel can be defined as [21]:

$$
f_{\gamma_{2}}(x)=\frac{N_{R}^{t}}{\Gamma\left(N_{D}\right)} \sum_{p=0}^{N_{R}^{t}-1}\left(\begin{array}{c}
N_{R}^{t}-1 \\
p
\end{array}\right)(-1)^{p} \exp \left(-\frac{(p+1) x}{\Omega_{2}}\right) \prod_{q=1}^{N_{D}-1}\left[\sum_{j_{q}=0}^{j_{q-1}}\left(\begin{array}{c}
j_{q-1} \\
j_{q}
\end{array}\right)\left(\frac{1}{q !}\right)^{j_{q}-j_{q+1}}\right] \Omega_{2}-\xi x^{\xi-1}
$$

where

$$
\left\{\begin{array}{c}
\xi=\beta_{q}+N_{D}, \\
\beta_{q}=\sum_{q=1}^{N_{D}-1} j_{q}, \\
j_{0}=p, \quad j_{N_{D}}=0, \text { and } \\
\Omega_{2}=\mathbf{E}\left[\left|h_{2(k)}\right|^{2}\right]
\end{array}\right.
$$

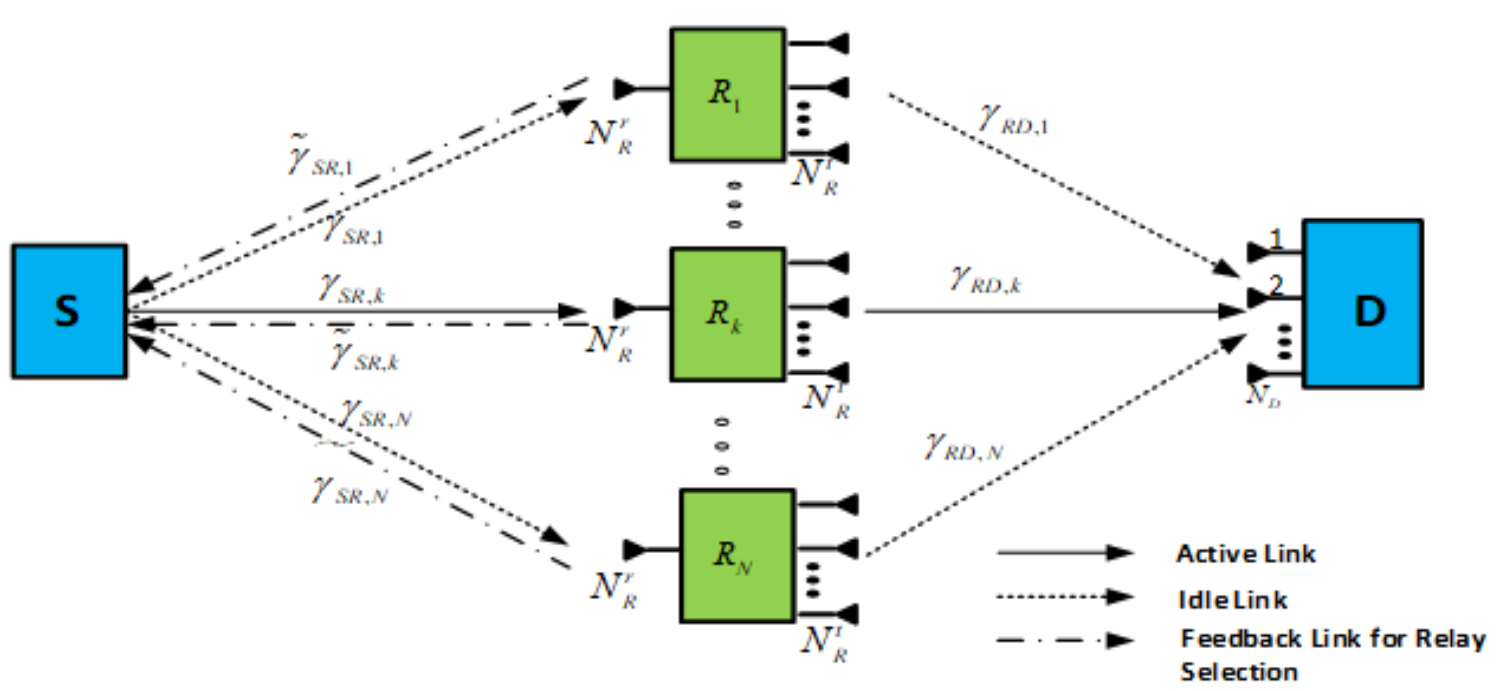

(a)

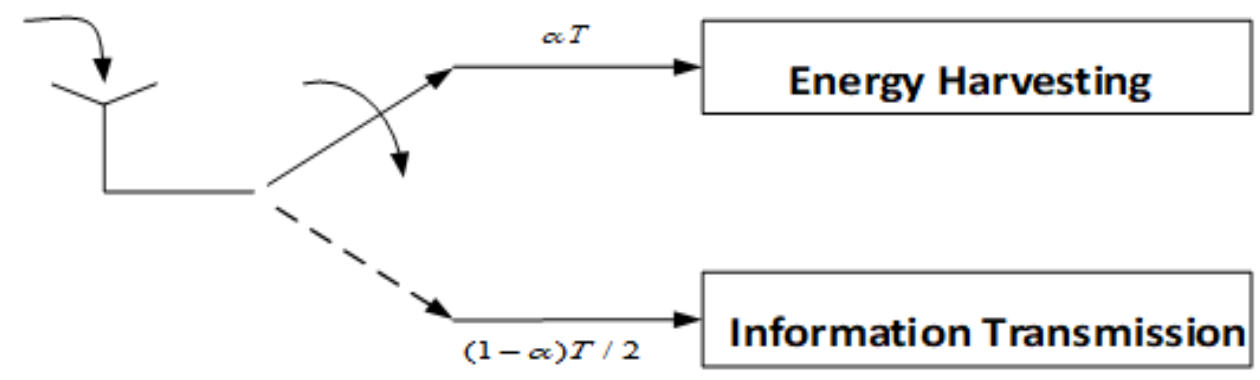

(b)

Figure 1: (a) Partial relay selection system model with energy harvesting (b) TSR protocol for power and information transfer at the relay

\section{STATISTICAL CHARACTERISTICS}

The CDF of the equivalent end-to-end instantaneous SNR at the destination for the concerned system can be expressed as [15]:

$$
\begin{aligned}
F_{e q}(\gamma) & =\operatorname{Pr}\left[\gamma_{1}<\frac{\psi \gamma \gamma_{2}+\gamma}{\phi \gamma_{1}} \mid \gamma_{2}>0\right] \\
& \triangleq \int_{0}^{\infty} F_{\gamma_{1}}\left(\frac{\psi \gamma \gamma_{2}+\gamma}{\phi \gamma_{2}}\right) f_{\gamma_{2}}\left(\gamma_{2}\right) d \gamma_{2}
\end{aligned}
$$

By substituting (7) and (8) into (10), the equivalent end-to-end instantaneous SNR for the system can be expressed as: 


$$
\begin{aligned}
F_{e q}(\gamma) & =1-\frac{k N_{R}^{t}}{\Gamma\left(N_{D}\right)} \sum_{n=0}^{k-1} \sum_{p=0}^{N_{R}^{t}-1}\left(\begin{array}{c}
N \\
k
\end{array}\right)\left(\begin{array}{c}
k-1 \\
n
\end{array}\right)\left(\begin{array}{c}
N_{R}^{t}-1 \\
p
\end{array}\right) \Omega_{2}^{-\xi} \frac{(-1)^{n+p}}{((N-k+n)+1)} \exp \left(-\frac{\Delta \psi \gamma}{\phi \Omega_{1}}\right) \\
& \times \prod_{q=1}^{N_{D}-1}\left[\sum_{j_{q}=0}^{j_{q-1}}\left(\begin{array}{c}
j_{q-1} \\
j_{q}
\end{array}\right)\left(\frac{1}{q !}\right)^{j_{q}-j_{q+1}}\right] \int_{0}^{\infty} \gamma_{2}{ }^{\xi-1} \exp \left(-\frac{(p+1) \gamma_{2}}{\Omega_{2}}\right) \exp \left(-\frac{\mu \gamma}{\phi \Omega_{1} \gamma_{2}}\right) d \gamma_{2}
\end{aligned}
$$

where

$$
\mu=\frac{(N-k+n+1) \gamma}{((N-k+n)(1-\rho)+1)}
$$

By converting the $\exp \left(-\frac{\mu \gamma}{\phi \Omega_{1} \gamma_{2}}\right)$ to Meijer-G function form using the identity defined [22, (11)] and invert the function using the identity detailed in $[23(8.2 .2(14)]$, then the integral part of (10) can be expressed as:

$$
v_{1}=\int_{0}^{\infty} \gamma_{2}^{\xi-1} \exp \left(-\frac{(p+1) \gamma_{R D}}{\Omega_{R D}}\right) G_{1,0}^{0,1}\left(\left.\frac{\phi \Omega_{2} \gamma_{2}}{\mu \gamma}\right|_{-} ^{1}\right) d \gamma_{2}
$$

By utilizing the integral identity defined in $[24,(7.813(1))]$, the (12) can be solved as:

$$
v_{1}=\left(\frac{\Omega_{2}}{(p+1)}\right)^{\xi} G_{2,0}^{0,2}\left(\left.\frac{\phi \Omega_{1} \gamma_{2}}{\mu \gamma(p+1)}\right|^{1-\xi, 1}-{ }^{1}\right)
$$

By putting (13) into (11), then the equivalent end-to-end instantaneous SNR for the system can be obtained as:

$$
\begin{gathered}
F_{e q}(\gamma)=1-\frac{k N_{R}^{t}}{\Gamma\left(N_{D}\right)} \sum_{n=0}^{k-1} \sum_{p=0}^{N_{R}^{t}-1}\left(\begin{array}{c}
N \\
k
\end{array}\right)\left(\begin{array}{c}
k-1 \\
n
\end{array}\right)\left(\begin{array}{c}
N_{R}^{t}-1 \\
p
\end{array}\right) \Omega_{2}^{-\xi} \frac{(-1)^{n+p}}{((N-k+n)+1)} \exp \left(-\frac{\mu \psi \gamma}{\phi \Omega_{1}}\right) \times \\
\prod_{q=1}^{N_{D}-1}\left[\sum_{\sum_{q}=0}^{j_{q-1}}\left(\begin{array}{c}
j_{q-1} \\
j_{q}
\end{array}\right)\left(\frac{1}{q !}\right)^{j_{q}-j_{q+1}}\right]\left(\frac{\Omega_{2}}{(p+1)}\right)^{\xi} G_{2,0}^{0,2}\left(\left.\frac{\phi \Omega_{1} \Omega_{R 2}}{\mu \gamma(p+1)}\right|^{1-\xi, 1}-\right.
\end{gathered}
$$

\section{PERFORMANCE ANALYSIS}

The performance analysis of the concerned system in terms of outage probability, ABER and throughput are presented in this section.

\subsection{Outage probability analysis}

The outage probability is an important measure for evaluating the performance of a wireless systems over fading channels. At a given transmission rate $R$ bits/s/Hz, the outage probability can be defined as [25, 26]:

$$
P_{\text {out }}=F_{e q}\left(\gamma_{t h}\right)
$$

where $\gamma_{t h}=2^{R}-1$ is the predetermined threshold SNR required to support the fixed transmission rate $R$ at the source in delay-limited transmission mode.

By substituting (14) into (15), the system outage probability can be expressed as:

$$
\begin{gathered}
P_{\text {out }}=1-\frac{k N_{R}^{t}}{\Gamma\left(N_{D}\right)} \sum_{n=0}^{k-1} \sum_{p=0}^{N_{R}^{t}-1}\left(\begin{array}{c}
N \\
k
\end{array}\right)\left(\begin{array}{c}
k-1 \\
n
\end{array}\right)\left(\begin{array}{c}
N_{R}^{t}-1 \\
p
\end{array}\right) \Omega_{2}^{-\xi} \frac{(-1)^{n+p}}{((N-k+n)+1)} \exp \left(-\frac{\mu \psi \gamma_{t h}}{\phi \Omega_{1}}\right) \times \\
\prod_{q=1}^{N_{D}-1}\left[\sum_{j_{q}=0}^{j_{q-1}}\left(\begin{array}{c}
j_{q-1} \\
j_{q}
\end{array}\right)\left(\frac{1}{q !}\right)^{j_{q}-j_{q+1}}\right]\left(\frac{\Omega_{2}}{(p+1)}\right)^{\xi} G_{2,0}^{0,2}\left(\left.\frac{\phi \Omega_{1} \Omega_{2}}{\mu \gamma_{t h}(p+1)}\right|^{1-\xi, 1}-\right.
\end{gathered}
$$

\subsection{Average bit error rate}

In this section, the ABER for the system is derived under the BPSK modulation and the ABER for the concerned system can be expressed as [15]:

$$
P_{b}=\frac{\delta}{2 \sqrt{2 \pi}} \int_{0}^{\infty} F_{e q}\left(\frac{x}{\omega}\right) \exp (-x / 2) x^{-\frac{1}{2}} d x
$$

where $\delta=1$ and $\omega=2$ for the BPSK modulation.

By substituting (11) into (17), the ABER for the system can be expressed as: 


$$
\begin{aligned}
P_{b}=\frac{\delta}{2 \sqrt{2 \pi}}\left[\int_{0}^{\infty} \exp (-x / 2) x^{-1 / 2} d x\right. & \\
& \left.\quad-\Xi_{1} \int_{0}^{\infty} x^{-1 / 2} \exp \left(-\left(\frac{\mu \psi}{\phi \omega \Omega_{1}}+\frac{1}{2}\right) t\right) G_{2,0}^{0,2}\left(\left.\frac{\phi \Omega_{1} \Omega_{2} \omega}{\mu(p+1) x}\right|^{1-\xi, 1}-1\right) d x\right]
\end{aligned}
$$

where

$$
\begin{aligned}
\Xi_{1} & =\frac{k N_{R}^{t}}{\Gamma\left(N_{D}\right)} \sum_{n=0}^{k-1} \sum_{p=0}^{N_{R}^{t}-1}\left(\begin{array}{c}
N \\
k
\end{array}\right)\left(\begin{array}{c}
k-1 \\
n
\end{array}\right)\left(\begin{array}{c}
N_{R}^{t}-1 \\
p
\end{array}\right) \Omega_{2}-\xi \frac{(-1)^{n+p}}{((N-k+n)+1)} \\
& \times \prod_{q=1}^{N_{D}-1}\left[\sum_{j_{q}=0}^{j_{q-1}}\left(\begin{array}{c}
j_{q-1} \\
j_{q}
\end{array}\right)\left(\frac{1}{q !}\right)^{j_{q}-j_{q+1}}\right]\left(\frac{\Omega_{2}}{(p+1)}\right)^{\xi}
\end{aligned}
$$

The first integral part of (18) can be solve by using the integral identity detailed in [24, $(3.326(2))]$ and the second integral can be solved by inverting the Meijer-G function using the identity defined in $[23,(8.2 .2(14))]$ as:

$$
v_{3}=\int_{0}^{\infty} x^{-1 / 2} \exp \left(-\left(\frac{\mu \psi}{\phi \omega \Omega_{1}}+\frac{1}{2}\right) t\right) G_{2,0}^{0,2}\left(\left.\frac{\mu(p+1) x}{\phi \Omega_{1} \Omega_{2} \omega}\right|_{\xi, 0} ^{-}\right) d x
$$

Then, by using the integral identity defined in $[24,(7.813(1))]$, the $v_{3}$ can be solved as:

$$
v_{3}=\left(\frac{2 \phi \omega \Omega_{1}}{2 \mu \psi+\phi \omega \Omega_{1}}\right)^{1 / 2} G_{0,2}^{2,0}\left(\frac{2 \mu(p+1)}{\Omega_{2}\left(2 \mu \psi+\phi \omega \Omega_{1}\right)} \mid \begin{array}{l}
1 / 2 \\
\xi, 0
\end{array}\right)
$$

Substituting $v_{3}$ into (18), the ABER for the TAS/MRC can be expressed as:

$$
\begin{aligned}
P_{b}= & \frac{\delta}{2 \sqrt{2 \pi}}\left[\frac{\Gamma(1 / 2)}{\sqrt{1 / 2}}-\frac{k N_{R}^{t}}{\Gamma\left(N_{D}\right)} \sum_{n=0}^{k-1} \sum_{p=0}^{N_{R}^{t}-1}\left(\begin{array}{c}
N \\
k
\end{array}\right)\left(\begin{array}{c}
k-1 \\
n
\end{array}\right)\left(\begin{array}{c}
N_{R}^{t}-1 \\
p
\end{array}\right) \Omega_{2}^{-\xi} \frac{(-1)^{n+p}}{((N-k+n)+1)} \times\right. \\
& \left.\prod_{q=1}^{N_{D}-1}\left[\sum_{j_{q}=0}^{j_{q-1}}\left(\begin{array}{c}
j_{q-1} \\
j_{q}
\end{array}\right)\left(\frac{1}{q !}\right)^{j_{q}-j_{q+1}}\right]\left(\frac{\Omega_{2}}{(p+1)}\right)^{\xi}\left(\frac{2 \phi \omega \Omega_{1}}{2 \mu \psi+\phi \omega \Omega_{1}}\right)^{1 / 2} G_{0,2}^{2,0}\left(\frac{2 \mu(p+1)}{\Omega_{2}\left(2 \mu \psi+\phi \omega \Omega_{1}\right)} \mid \begin{array}{c}
1 / 2 \\
\xi, 0
\end{array}\right)\right]
\end{aligned}
$$

\subsection{Throughput analysis}

In this paper, the delay-limited transmission mode is employed for the concerned system and the throughput is obtained by determining the outage probability of the system at a fixed source transmission rate $R$ bits $/ \mathrm{s} / \mathrm{Hz}$. Therefore, at a fixed transmission rate with effective communication time of $(1-\alpha) T / 2$ from the source to destination, the throughput $\tau$ can be expressed as [7]:

$$
\tau=\left(1-P_{\text {out }}\right) R \frac{(1-\alpha) T / 2}{T} \triangleq \frac{R}{2}\left(1-P_{\text {out }}\right)(1-\alpha)
$$

By putting (16) into (23), the throughput for the concerned system can be expressed as:

$$
\begin{aligned}
& \tau=\frac{(1-\alpha) R k N_{R}^{t}}{2 \Gamma\left(N_{D}\right)} \sum_{n=0}^{k-1} \sum_{p=0}^{N_{R}^{t}-1}\left(\begin{array}{c}
N \\
k
\end{array}\right)\left(\begin{array}{c}
k-1 \\
n
\end{array}\right)\left(\begin{array}{c}
N_{R}^{t}-1 \\
p
\end{array}\right) \Omega_{2}^{-\xi} \frac{(-1)^{n+p}}{((N-k+n)+1)} \exp \left(-\frac{\mu \psi \gamma_{t h}}{\phi \Omega_{1}}\right) \times \\
& \prod_{q=1}^{N_{D}-1}\left[\sum_{j_{q}=0}^{j_{q-1}}\left(\begin{array}{c}
j_{q-1} \\
j_{q}
\end{array}\right)\left(\frac{1}{q !}\right)^{j_{q}-j_{q+1}}\right]\left(\frac{\Omega_{2}}{(p+1)}\right)^{\xi} G_{2,0}^{0,2}\left(\left.\frac{\phi \Omega_{1} \Omega_{2}}{\Delta \gamma_{t h}(p+1)}\right|^{1-\xi, 1} \begin{array}{c}
- \\
-
\end{array}\right)
\end{aligned}
$$

\section{NUMERICAL RESULTS AND DISCUSSIONS}

In this section, the numerical results for the system outage probability, ABER, and throughput are presented based on the derived analytical closed form expression presented in (16), (22), and (24) respectively. Unless specified, the system parameters adopted are set to be: $R=2 \mathrm{bits} / \mathrm{s} / \mathrm{Hz}, \Omega_{1}=1$, $\Omega_{2}=1, \eta=1, \vartheta_{1}=2, \vartheta_{2}=3$, and $\gamma_{T}=20 \mathrm{~dB}$. Two selection scenarios are considered as follows: worst relay selection when $k=1$ and best relay selection when $k=N$

In Figure 2, the effect of S-to-R distance on the concerned system under different relay selection scenarios is demonstrated The result shows that as $d_{1}$ increases, both the energy harvested and received signal at the relay nodes become weaker causing the system outage probability to be degraded. However, beyond an optimum distance of $d_{1}$ where the distance between the relay and destination becomes smaller, the system outage probability starts decreasing even with less transmit power for the relay to transmit 
information. Irrespective of this, the best relay selection offers the system better performance than the worst relay selection.

The outage performance of the concerned system under different values of channel correlation coefficient at different relay distant is illustrated in Figure 3. The results show that as the transmit SNR increase the better the system outage probability. In addition, it can be depicted that the increase in correlation coefficient the better the system outage performance. However, the as the relays location far from the source, the worse the outage performance becomes due to less transmit power received at the relay node for information transmission. The results also indicated the simulation result perfectly match with the analytical result which proves the correctness of our derived expressions.

The effect of channel correlation coefficient on the ABER of the system is illustrated in Figure 4 under different number of antenna at the destination. It can be clearly seen that increase in the number of destination antenna leads to reduction in the system error performance. It is also depicted from the results that the higher the channel correlation the better the system performance. The results also indicate that the analytical results are well agreed with the simulation results which shows the accuracy of our derivation.

The error performance as a function of energy harvesting time is presented in Figure 5 under number of relay selection. It can be shown that as the energy harvesting time increases the better the system error performance. This is owing to the large amount of time available for relay nodes to replenished energy for information transmission. Also, the increase in the number of relay selection for transmission significantly improves the system error performance.

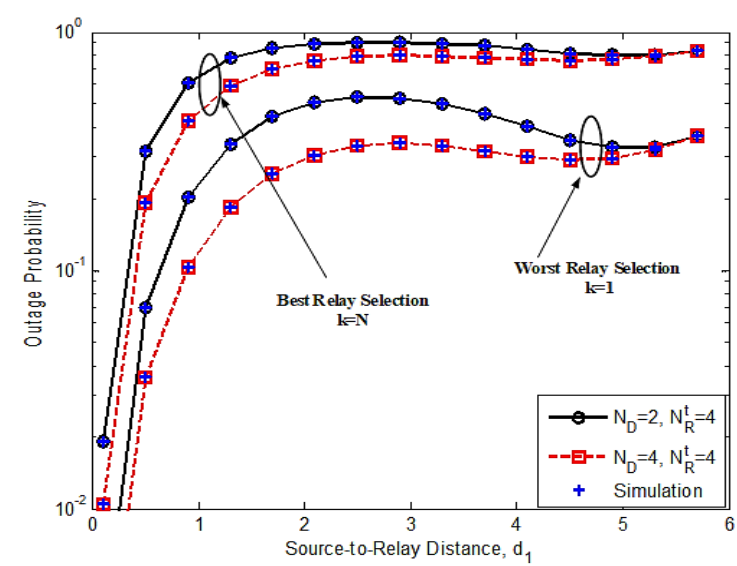

Figure 2. Outage probability vs the S-to-R distance under different number of $N_{D}$ at $\alpha=0.5$,

$$
\rho=0.6, \text { and } d_{2}=6-d_{1}
$$

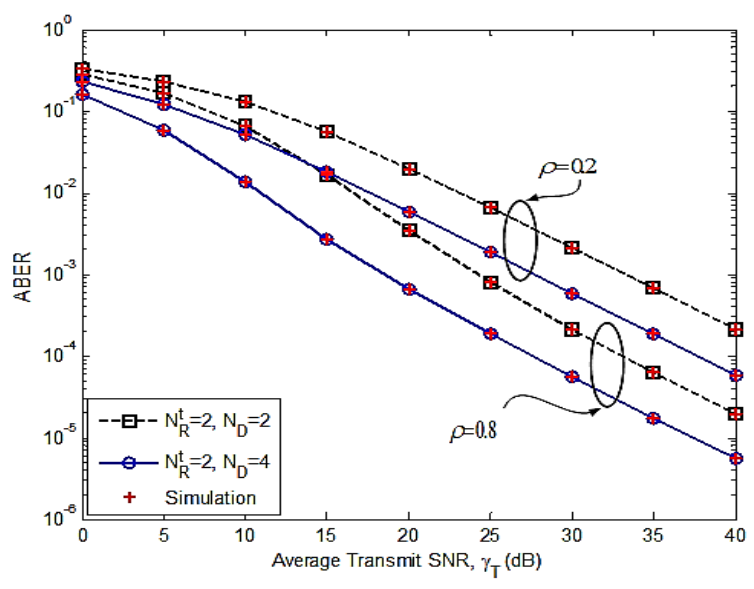

Figure 4. Impact of number of antenna at the destination $N_{D}$ on the system ABER under different correlation coefficients at $\alpha=0.6, k=N=4$

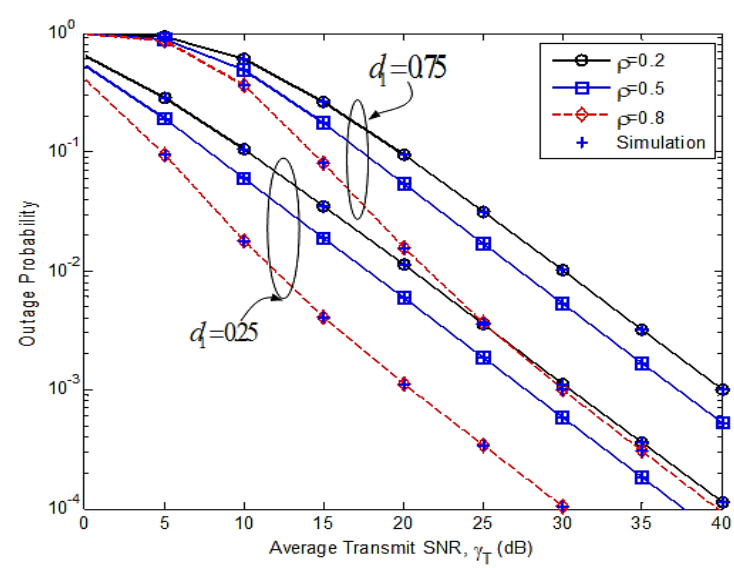

Figure 3. Effect of correlation coefficient on the system outage probability under different S-to-R distance

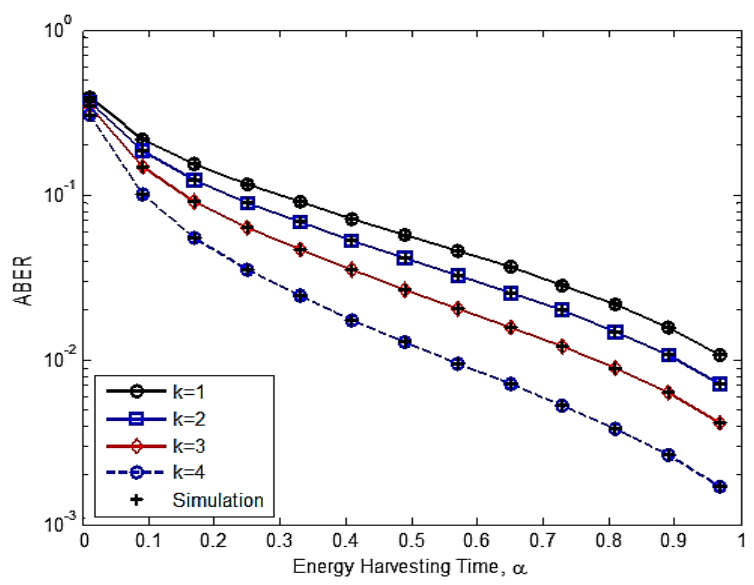

Figure 5. ABER performance of the system under different relay selection at $N=4, \rho=0.6, N_{D}=2, N_{R}^{t}=2$ 
In Figure 6, the throughput performance of the system as a function of energy harvesting time is depicted under different average transmit SNR. The results show that the increase in the source transmit power, the higher the system throughput because larger energy is harvested at relay nodes. It can be deduced from the results that, at high transmit power, the optimum time is very short for energy harvesting and large time is used to decode information. Compare with the lower transmit power. The analytical results are perfectly matched with the simulation results showing the accuracy of the derived expression.

The throughput as a function of relay distance from the source under different number of antenna at the destination is demonstrated in Figure 7. The results indicate that the system performance is significantly improved with the increase in the number of antenna at the destination. It can be seen from the results that at small values $d_{1}$, the system throughput becomes degraded due to larger distance between the relay and the destination, and the outage probability is high at the regime. Thus, at the optimum relay location, the system performance starts increasing snice the smaller value of $d_{2}$ result into higher system throughput with better system outage. As expected, the results depict that the system has better performance under the best relay selection compare with worst relay selection.

The throughput performance of the system at different channel correlation coefficient and $N_{D}$ is depicted in Figure 8. It can be seen from the results that higher throughput performance can be achieved with the best relay selection at high SNR. At low and high SNR, the throughput becomes saturated which shows that the outage probability has higher and lower values respectively. In addition, the results also prove that increase in the correlation coefficient significantly improve the system performance. The simulation results perfect matched with the analytical results which indicate the correctness of our derivation.

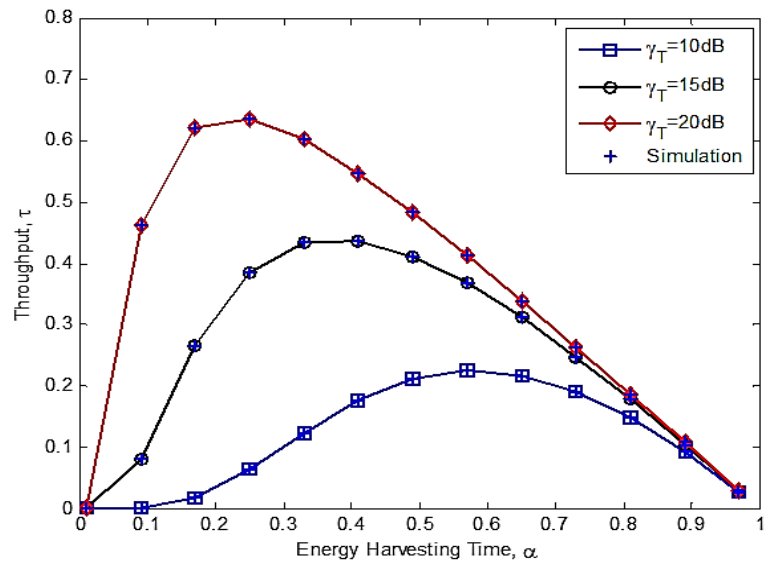

Figure 6. Throughput versus the energy harvesting time under different average transmit SNR at $\rho=0.6$, $N_{D}=2, N_{R}^{t}=4, k=N=4$

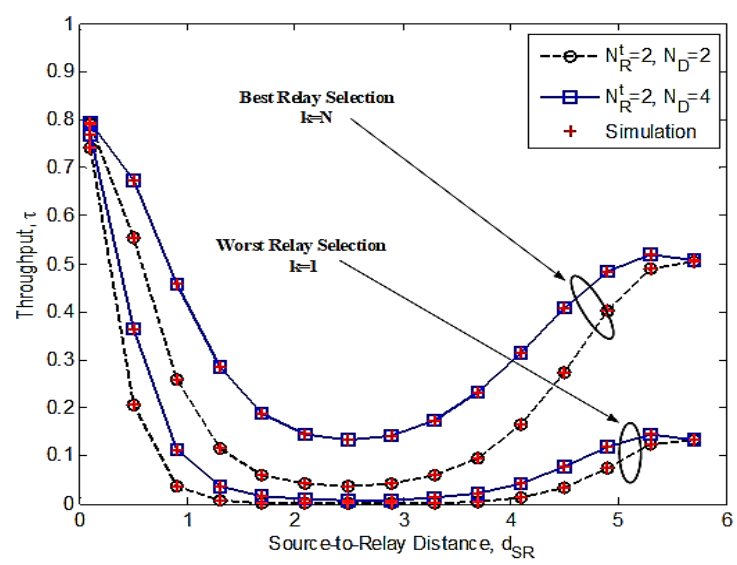

Figure 7. Effect of S-to-R distance on the system throughput under different number of $N_{D}$ for different relay selection scenarios when $\alpha=0.2, \rho=0.6$, and $d_{2}=6-d_{1}$

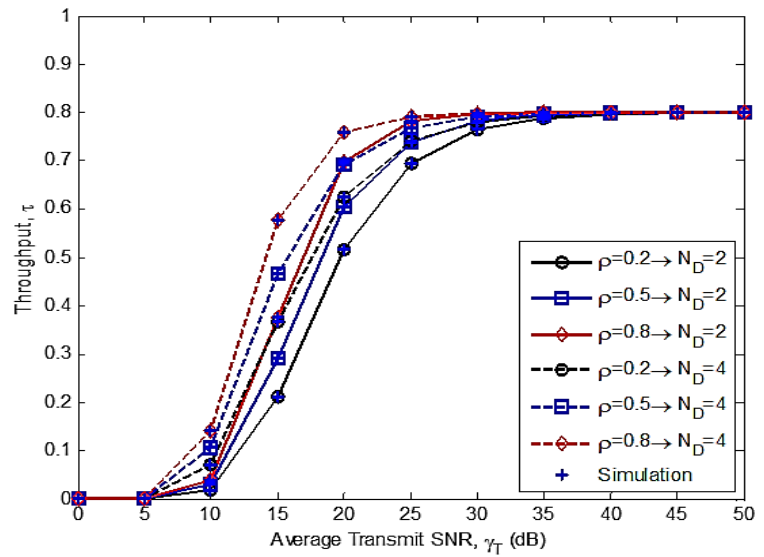

Figure 8. Performance system throughput under different correlation coefficient and $N_{D}$ when $\alpha=0.2$ at $k=N=4$ 


\section{CONCLUSION}

The performance of energy harvesting based PRS cooperative system with TAS and outdated channel state is presented. Based on the TS protocol, the analytical closed form expression for the system outage probability and ABER are obtained. Through the outage probability, the system throughput for delay-limited transmission mode is determined. These exact expressions are verified by the Monte Carlo simulation. The results illustrated that the increase in channel coefficient significantly improve the system performance. In addition, the system offers better performance with the increase in number of the destination receive antenna. Also, the result shows that system outage probability, ABER and throughput performance improved with increasing the number of relays or the values of the correlation coefficient.

\section{REFERENCES}

[1] K. Zhu, F. Wang, S. Li, F. Jiang, and L. Cao, "Relay Selection for Cooperative Relaying in Wireless Energy Harvesting Networks," Proceedings of IOP Conference Series: Earth and Environmental Science, vol. 108, no. 5 , pp. $1-8,2018$.

[2] Y. Gu and S. Aïssa, "RF-based energy harvesting in decode-and-forward relaying systems: Ergodic and outage capacities," IEEE Transactions on Wireless Communications, vol. 14, no. 11, pp. 6425-6434, 2015.

[3] C. Yin, N.-P. Nguyen, E. Garcia-Palacios, X. N. Tran, and T. Le-Tien, "Secure energy harvesting communications with relay selection over nakagami-m fading channels," Mobile Networks and Applications, vol. 23, no. 6, pp. 1555-1562, 2017.

[4] A. Andrawes, R. Nordin, and M. J. E. Ismail, "Wireless Energy Harvesting with Cooperative Relaying under the Best Relay Selection Scheme," Energies, vol. 12, no. 5, pp. 1-22, 2019.

[5] C. Zhong, H. A. Suraweera, G. Zheng, I. Krikidis, and Z. Zhang, "Wireless information and power transfer with full duplex relaying," IEEE Transactions on Communications, vol. 62, no. 10, pp. 3447-3461, 2014.

[6] K.-H. Liu and Te-Lin Kung, "Performance improvement for RF energy-harvesting relays via relay selection," IEEE Transactions on Vehicular Technology, vol. 66, no. 9, pp. 8482-8494, 2017.

[7] A. A. Nasir, X. Zhou, S. Durrani, and R. A. Kennedy, "Relaying protocols for wireless energy harvesting and information processing," IEEE Transactions on Wireless Communications, vol. 12, no. 7, pp. 3622-3636, 2013.

[8] V. P. Tuan and H. Y. Kong, "Impact of residual transmit RF impairments on energy harvesting relay selection systems," International Journal of Electronics , vol. 104, no. 6, pp. 928-941, 2017.

[9] C. Yin, X. He, N.-P. Nguyen, and E. Garcia-Palacios, "Secure Energy Harvesting Communications with Partial Relay Selection over Nakagami-m Fading Channels," Proceedings of International Conference on Industrial Networks and Intelligent Systems, pp. 100-110, 2017.

[10] K. O. Odeyemi and P. Owolawi, "Impact of transmission techniques in asymmetric RF/FSO system over Nakagami-m and gamma-gamma fading channels with pointing errors," International Journal of Communication Systems, vol. 32, no. 3, pp. 1-20, 2019.

[11] M. Soysa, H. A. Suraweera, C. Tellambura, and H. K. Garg, "Amplify-and-forward partial relay selection with feedback delay," Proceedings of 2011 IEEE Wireless Communications and Networking Conference, pp. 1304-1309, 2011.

[12] M. Soysa, H. A. Suraweera, C. Tellambura, and H. K. Garg, "Partial and opportunistic relay selection with outdated channel estimates," IEEE Transactions on Communications, vol. 60, no. 3, pp. 840-850, 2012.

[13] H. A. Suraweera, M. Soysa, C. Tellambura, and H. K. Garg, "Performance analysis of partial relay selection with feedback delay," IEEE Signal Processing Letters, vol. 17, no. 6, pp. 531-534, 2010.

[14] O. S. Badarneh, F. S. Almehmadi, I. S. Ansari, and X. Yang, "Wireless energy harvesting in cooperative decodeand-forward relaying networks over mixed generalized $\eta-\mu$ and $\kappa-\mu$ fading channels," Transactions on Emerging Telecommunications Technologies, vol. 29, no. 2, pp. 1-12, 2018.

[15] D.-D. Tran, D.-B. Ha, H.-V. Tran, and G. Kaddoum, "Wireless information transfer in relay networks with energy harvesting over non-identical channels," Proceedings of 2015 International Conference on Communications, Management and Telecommunications, pp. 172-177, 2015.

[16] K. M. Rabie, A. Salem, E. Alsusa, and M.-S. Alouini, "Energy-harvesting in cooperative AF relaying networks over log-normal fading channels," Proceedings of 2016 IEEE International Conference on Communications, pp. 1-7, 2016.

[17] P. V. T. Anh, V. N. Q. Bao, and K. N. Le, "On the performance of wireless energy harvesting TAS/MRC relaying networks over Nakagami-m fading channels," Proceedings of 2016 3rd National Foundation for Science and Technology Development Conference on Information and Computer Science, pp. 1-5, 2016.

[18] Y. Luo, J. Zhang, and K. B. Letaief, "Relay selection for energy harvesting cooperative communication systems," Proceedings of 2013 IEEE Global Communications Conference, pp. 2514-2519, 2013.

[19] V. N. Q. Bao and N. T. Van, "Incremental relaying networks with energy harvesting relay selection: Performance analysis," Transactions on Emerging Telecommunications Technologies, vol. 29, no. 12, pp. 1-22, 2018.

[20] H. Y. Kong, "Wireless Information and Power Transfer in Kth Best Relay Selection Systems with Energy Beamforming over Nakagami-m Fading Channels," Wireless Personal Communications, vol. 97, no. 3, pp. 4229-4249, 2017. 
[21] N. Yang, P. L. Yeoh, M. Elkashlan, R. Schober, and I. B. Collings, "Transmit antenna selection for security enhancement in MIMO wiretap channels," IEEE Transactions on Communications, vol. 61, no. 1, pp. 144-154, 2013.

[22] V. Adamchik and O. Marichev, "The algorithm for calculating integrals of hypergeometric type functions and its realization in REDUCE system," Proceedings of Proceedings of the international symposium on Symbolic and algebraic computation, pp. 212-224, 1990.

[23] Y. A. Brychkov, O. Marichev, and A. Prudnikov, "Integrals and Series, vol 3: more special functions," ed: Gordon and Breach science publishers, 1986.

[24] I. Gradshteyn, I. Ryzhik, and R. H. Romer, "Tables of integrals, series, and products," ed: AAPT, 1988.

[25] Y. Huang, J. Wang, P. Zhang, and Q. Wu, "Performance analysis of energy harvesting multi-antenna relay networks with different antenna selection schemes," IEEE Access, vol. 6, pp. 5654-5665, 2018.

[26] K. O. Odeyemi and P. A. Owolawi, "Selection combining hybrid FSO/RF systems over generalized induced-fading channels," Optics Communications, vol. 433, pp. 159-167, 2019.

\section{BIOGRAPHIES OF AUTHORS}
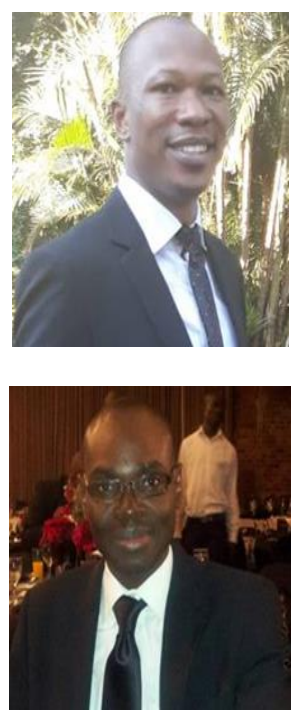

Kehinde Oluwasesan Odeyemi received his B.Tech. degree in Electronic Engineering from Ladoke Akintola University of Technology Ogbomosho, Oyo State, Nigeria, in 2008. He later obtained an M.Eng. Degree in the same field from the Federal University of Technology, Akure in 2012. He received his Ph.D. degree in Electronic Engineering at the University of KwaZulu-Natal, Durban, South Africa in 2018. In 2012, he joined the department of Electrical and Electronic Engineering, University of Ibadan, Nigeria. He is a member of The Council for the Regulation of Engineering in Nigeria (COREN). He has written several research articles and his research interests are in the antenna design, optical wireless communications, diversity combining techniques and MIMO systems.

Pius Adewale Owolawi received his B.Tech. degree in Physics/Electronics from the Federal University of Technology, Akure, in 2001. He then obtained a M.Sc. and Ph.D. degrees in Electronic Engineering from the University of KwaZulu-Natal in 2006 and 2010, respectively. He holds several industry certifications such as CCNA, CCNP, CWNP, CFOA, CFOS/D and MCITP. Member of several professional bodies like SAIEE, IEEE, and SA AMSAT. In 2007, he joined the faculty of the Engineering, Department of Electrical Engineering at Mangosuthu University of Technology, South Africa. Thereafter, in 2017, he joined the department of Computer Systems Engineering, Tshwane University of Technology, Pretoria, South Africa where is currently the head of department. His research interests are in the computational of Electromagnetic, modeling of radio wave propagation at high frequency, fiber optic communication, radio planning and optimization techniques and renewable energy. He has written several research articles and serves as a reviewer for many scientific journals. 